\title{
Amiodarone Prophylaxis for Atrial Fibrillation after Coronary Bypass-12 Years Follow-Up
}

\author{
Cecilie Enevoldsen 1,2, Britt Borregaard1,2, Sara Schoedt Riber1,2, Lars Schoedt Riber 1,2* \\ ${ }^{1}$ Department of Cardiothoracic and Vascular Surgery, Odense University Hospital, Odense, Denmark \\ ${ }^{2}$ Faculty of Health Sciences, University of Southern Denmark, Odense, Denmark \\ Email: ${ }^{\star}$ lars.riber@rsyd.dk
}

How to cite this paper: Enevoldsen, C., Borregaard, B., Riber, S.S. and Riber, L.S. (2020) Amiodarone Prophylaxis for Atrial Fibrillation after Coronary Bypass-12 Years Follow-Up. Open Journal of Thoracic Surgery, 10, 19-31.

https://doi.org/10.4236/ojts.2020.101003

Received: December 13, 2019

Accepted: January 28, 2020

Published: January 31, 2020

Copyright $\odot 2020$ by author(s) and Scientific Research Publishing Inc. This work is licensed under the Creative Commons Attribution International License (CC BY 4.0).

http://creativecommons.org/licenses/by/4.0/

\section{(c) (i) Open Access}

\begin{abstract}
Objectives: Postoperative atrial fibrillation (POAF) is a common complication after cardiac surgery and might be avoided with amiodarone prophylaxis. We investigated the long-term effect of prophylactic amiodarone, in patients undergoing isolated, elective coronary artery bypass grafting (CABG), as well as the long-term prognosis of patients with POAF. Methods: We retrospectively analysed patients included in the randomized, control trial RASCABG allocated to either placebo or amiodarone as POAF prophylaxis. Several outcomes were evaluated using national databases. Patients were stratified and compared by prophylactic groups and rhythm. Cox proportional hazard analysis was used for multivariable analysis. Kaplan-Meier survival curves were constructed to assess the probability of survival and cumulative incidence curves. Results: Prophylactic amiodarone was not related to long-term side effects in comparison to placebo. Patients who developed POAF, were more likely to develop late AF, and had a worse overall survival. There was no difference in the overall survival between prophylactic groups. In the competing risk analysis, amiodarone and POAF was not associated with an increased risk of readmission, but POAF was associated with an increased risk of late AF HR 2.28 [95\% CI 1.15; 4.55]. In the multivariable analysis, amiodarone was not associated with late $\mathrm{AF}$, readmission or long-term mortality. Further, no association was found between POAF and late AF, readmission or long-term mortality. Conclusion: Amiodarone is a safe drug for the prevention of POAF in the RASCABG-setting. POAF is related to long-term worse prognosis regarding late $\mathrm{AF}$ and mortality.
\end{abstract}

\section{Keywords}

Coronary Artery Bypass Grafting, Atrial Fibrillation, Postoperative Care, Pharmacology 


\section{Introduction}

Postoperative atrial fibrillation (POAF) is a common complication following cardiac surgery with a reported incidence of $15 \%-80 \%$, depending on patient characteristics, surgical procedure, surveillance methods and definition of atrial fibrillation (AF) [1] [2] [3] [4]. Usually, POAF develops within the first four days after surgery, with a peak incidence on the second postoperative day [1] [2]. Although the majority of events are uncomplicated and self-limiting, POAF has been associated with a significant increase in hospital stay, morbidity and mortality [2] [4] [5] [6] [7]. The underlying pathophysiology and aetiology of POAF remain unclear, although studies have reported causes of a multifactorial nature, involving inflammation, oxidative stress, electrolyte disturbances, atrial dilation, age-related fibroses, pre-existing structural remodelling and increased sympathetic tone postoperatively [1] [3] [4]. Several possible risk factors for POAF has been found including age, male sex, obesity, combination of coronary artery bypass grafting $(\mathrm{CABG})$ and valve surgery or isolated valve surgery, prior $A F$ or arrhythmia, chronic obstructive pulmonary disease (COPD), congestive heart failure, left atrial enlargement and left ventricular dysfunction [1] [2] [3] [4].

Studies have attempted to determine whether antiarrhythmic drugs already being used in the primary treatment of AF, could prevent the risk of POAF [1] [8] [9] [10] [11]. In 2004-2005, the Danish randomized control trial, RASCABG, was conducted, in which 250 patients undergoing elective, solitary CABG were allocated to receive either amiodarone or placebo, as prophylaxis for POAF with a follow-up of 30-days [12]. As hypothesized, patients in the intervention group experienced a significant reduction in risk of $\mathrm{AF}$ at $14 \%$, with a number needed to treat at 6.9 [12]. No severe side effects were found during the 30 -days follow-up [12]. To our knowledge, no studies have yet investigated the long-term effect or side-effects of amiodarone on POAF following CABG.

Thus, the objectives of this 12-year follow-up study among patients receiving prophylactic amiodarone or placebo was to: Describe differences in baseline characteristics, the frequency of stroke and heart failure, readmission, late AF, long-term all-cause mortality and long-term side effects, and investigate associations between amiodarone and placebo and the risk of readmission, developing late AF, long-term all-cause mortality and cardiovascular mortality. And, among patients developing POAF to describe differences in baseline characteristics, the frequency of stroke and heart failure, readmission, late $\mathrm{AF}$ and long-term all-cause mortality, and to investigate the associations between POAF and the risk of readmission, developing late $\mathrm{AF}$, long-term all-cause mortality and cardiovascular mortality.

\section{Materials and Methods}

\subsection{Study Population and Data Extraction}

We retrospectively analysed 12-year follow-up data on 250 patients who were included in the randomized control trial, RASCABG [12]. Inclusion criteria of 
the original study were elective CABG, age older than 18 years, willingness to be randomized, and provision of informed consent. Exclusion criteria were other types of heart surgery, previous heart surgery, resting heart rate of less than 40 beats/min, atrial-ventricular blockage of any degree, known AF or atrial flutter, hepatic dysfunction (alanine aminotransferase level more than twice the upper normal limit), hyperthyroidism, pregnancy, breast-feeding, or known adverse reactions to amiodarone [12]. No adverse events were found in the primary study. In the current study, we furthermore excluded patients with protocol violation.

\subsection{Variables and Definitions}

Baseline data were extracted from the original dataset and the Western Denmark Heart Registry [13]. Data on follow-up were obtained from the Danish Register of Causes of Death, the Western Denmark Heart Registry, and the Danish National Patient Registry [13] [14] [15]. Follow-up data included heart failure, late AF, readmission, stroke, long-term side effects and, long-term all-cause mortality and cardiovascular mortality. Unless specified otherwise, heart failure, late $\mathrm{AF}$, readmission and stroke, will be referred to as HARS (Heart failure, late Atrial fibrillation, Readmission and Stroke). Late AF, stroke, heart failure, long-term side effects, long-term all-cause mortality and cardiovascular mortality were evaluated as binary variables defined by diagnostic codes, classified by the ICD-10 classification. Diagnostic codes were clustered into categories e.g. "cancer", and subcategories e.g. "lung cancer", and converted into variables. Diagnostic codes that only occurred once and could not be categorised with others, were excluded. For the variable of late AF, we included diagnostic codes for atrial flutter, $\mathrm{AF}$ and unspecified atrial flutter or fibrillation. Late AF was defined as atrial fibrillation during the follow-up period, occurring after the 30. postoperative day. Long-term mortality was divided into all-cause mortality, defined as death of any cause during the follow-up period, and cardiovascular mortality defined as death due to cardiovascular diseases. Readmission was evaluated as the number of patients readmitted during the follow-up period. Time to readmission and $\mathrm{AF}$, were defined as the time interval from the surgery date, to the first readmission and AF during the follow-up period, respectively.

Data access and processing were approved by Danish Data Protection Agency (2017-41-5275) and the Danish Patient Safety Authority (3-3013-2374/1).

\subsection{Statistical Methods}

Numerical variables were tested for normality of distribution using the Shapiro-Wilk test, and reported as median and quartiles (interquartile range (IQR) from 25th to 75 th quartile) or mean and standard deviation (SD). For categorical variables, the number and percentage of subjects in each category was reported. Baseline differences and differences in stroke, heart failure, readmission, late AF, long-term all-cause mortality and long-term side effects among groups were tested using Students t-test or Mann-Whitney U test, for numerical variables, and Pear- 
son's $\chi^{2}$ test of independence or Fischer's exact test for categorical variables.

We analysed prophylactic groups (intervention vs. placebo) and subgroups (POAF vs. sinus rhythm) separately.

Cox multivariable proportional hazard regression analysis, adjusted for potential confounders, was used to investigate the association between 1) amiodarone and readmission, late $\mathrm{AF}$, long-term all-cause mortality and cardiovascular mortality 2) POAF and readmission, late AF, long-term all-cause mortality and cardiovascular mortality. Results were reported as hazard ratios (HR) with 95\% confidence intervals (CI).

Kaplan-Meier survival curves were used to estimate long-term all-cause mortality for prophylactic groups and subgroups respectively, during the follow-up period. Survival time was determined from the date of surgery to date of event or 12 years, having survived the follow-up. Differences in mortality rates between prophylactic groups and subgroups were compared using Log-rank test.

The time-to-event data included time to first AF and time to the first readmission from the date of surgery. Cumulative incidence function (CIF) was used in a Fine and Gray Proportional Hazard Regression Model to investigate the time to event data, in the presence of competing events [16].

All p-values are two-tailed, and a p-value $<0.05$ was considered as statistically significant. All the data were analysed using Stata Statistical Software 15.0 (StataCorp LLC, College Station, TX).

\section{Results}

A total of 196 patients from the RASCABG trial [12] were eligible and included in the follow-up study as two patient were lost to follow-up in Figure 1.

\subsection{Baseline Characteristics}

In the baseline characteristics, triple vessel disease was overrepresented in the amiodarone group, and the number of grafts used per case, as the use of two grafts was more frequent in the placebo group in Table 1 . Patients who developed

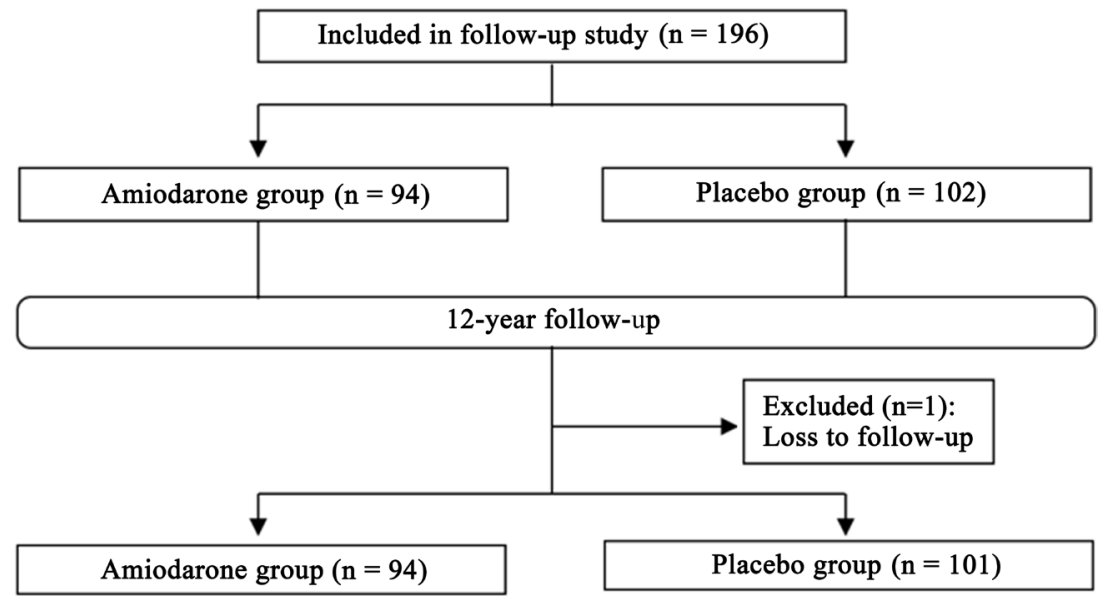

Figure 1. Flowchart of study population. 
Table 1. Baseline Characteristics

\begin{tabular}{|c|c|c|c|c|c|c|c|}
\hline & \multirow{2}{*}{ All } & \multicolumn{2}{|c|}{ Prophylactic Groups } & \multirow{2}{*}{$p$-Value } & \multicolumn{2}{|c|}{ Subgroups } & \multirow{2}{*}{$p$-Value } \\
\hline & & Amiodarone & Placebo & & POAF & NO POAF & \\
\hline \multicolumn{8}{|l|}{ Characteristics } \\
\hline $\mathrm{N}(\%)$ & $196(100)$ & $94(48)$ & $102(52)$ & N/A & $45(23)$ & $151(77)$ & N/A \\
\hline Age (median, IQR) & $65(59 ; 71)$ & $66(60 ; 72)$ & $65(58 ; 70)$ & 0.605 & $69(64 ; 74)$ & $64(57 ; 70)$ & $0.001^{*}$ \\
\hline Sex (male, n (\%)) & $166(85)$ & $83(88)$ & $83(81)$ & 0.179 & $40(89)$ & $126(83)$ & 0.373 \\
\hline Current smoker (n (\%)) & $51(26)$ & $26(28)$ & $25(25)$ & 0.616 & $12(27)$ & $39(26)$ & 0.910 \\
\hline Diabetes mellitus (n (\%)) & $48(25)$ & $23(25)$ & $25(25)$ & 0.995 & $13(29)$ & $35(23)$ & 0.434 \\
\hline Hypertension (n (\%)) & $127(65)$ & $61(65)$ & $66(65)$ & 0.978 & $28(62)$ & $99(66)$ & 0.680 \\
\hline Hypercholesterolemia (n (\%)) & $136(69)$ & $64(68)$ & $72(71)$ & 0.704 & $27(60)$ & $109(72)$ & 0.120 \\
\hline Family history of IHD (n (\%)) & $93(47)$ & $41(48)$ & $52(57)$ & 0.238 & $17(45)$ & $76(54)$ & 0.296 \\
\hline History of CNS events (n (\%)) & $46(24)$ & $21(23)$ & $25(28)$ & 0.414 & $12(31)$ & $34(24)$ & 0.413 \\
\hline COPD (n (\%)) & $13(7)$ & $9(10)$ & $4(4)$ & 0.112 & $6(13)$ & $7(5)$ & 0.079 \\
\hline \multicolumn{8}{|l|}{ NYHA Class (n (\%)) } \\
\hline NYHA Class I & $56(29)$ & $27(29)$ & $29(28)$ & 0.964 & $12(27)$ & $44(29)$ & 0.747 \\
\hline NYHA Class II & $92(50)$ & $44(47)$ & $48(47)$ & 0.972 & $17(38)$ & $75(50)$ & 0.161 \\
\hline NYHA Class III & $46(24)$ & $23(25)$ & $23(23)$ & 0.751 & $15(33)$ & $31(21)$ & 0.075 \\
\hline NYHA Class IV & ** & ** & ** & 0.498 & ** & $* *$ & 0.407 \\
\hline \multicolumn{8}{|l|}{ CCS Class (n (\%)) } \\
\hline CCS Class I & $40(20)$ & $19(20)$ & $21(21)$ & 0.948 & $13(29)$ & $27(18)$ & 0.108 \\
\hline CCS Class II & $94(48)$ & $47(46)$ & $47(50)$ & 0.583 & $17(38)$ & $77(51)$ & 0.119 \\
\hline CCS Class III & $49(25)$ & $21(22)$ & $28(28)$ & 0.409 & $12(27)$ & $37(25)$ & 0.769 \\
\hline CCS Class IV & $13(7)$ & $7(7)$ & $6(6)$ & 0.660 & $* *$ & $10(7)$ & 1.000 \\
\hline Previous MI (n (\%)) & $93(47)$ & $45(48)$ & $48(47)$ & 0.909 & $18(40)$ & $75(50)$ & 0.254 \\
\hline Previous PCI (n (\%)) & $17(9)$ & $7(7)$ & $10(10)$ & 0.558 & ** & $15(10)$ & 0.369 \\
\hline Preoperative use of $\beta$-blockers (n (\%)) & $170(87)$ & $81(86)$ & $89(87)$ & 0.823 & $38(84)$ & $132(87)$ & 0.606 \\
\hline Preoperative use of statins (n (\%)) & $173(88)$ & $81(86)$ & $92(90)$ & 0.382 & $38(84)$ & $135(89)$ & 0.364 \\
\hline EF (median, IQR) & $56(49 ; 60)$ & $55(48 ; 60)$ & $56(50 ; 60)$ & 0.857 & $56(45 ; 60)$ & $57(50 ; 60)$ & 0.392 \\
\hline Creatinine $(\mu \mathrm{mol} / \mathrm{L})$ (median, IQR) & $88(75 ; 99)$ & $89(75 ; 99)$ & $87(76 ; 99)$ & 0.818 & $91(79 ; 106)$ & $88(75 ; 98)$ & 0.106 \\
\hline BMI (mean, SD) & $27.52(4.14)$ & $27.50(4.06)$ & $27.54(4.23)$ & 0.943 & $28.24(4.37)$ & $27.30(4.06)$ & 0.184 \\
\hline EuroSCORE I (median, IQR) & $2(1 ; 4)$ & $3(1 ; 4)$ & $2(1 ; 4)$ & 0.210 & $3(2 ; 5)$ & $2(1 ; 4)$ & $0.009^{*}$ \\
\hline \multicolumn{8}{|l|}{ Vessels affected (No. (n (\%)) } \\
\hline 1 & ** & ** & $* *$ & 0.498 & ** & $* *$ & 0.407 \\
\hline 2 & $20(10)$ & $6(6)$ & $14(14)$ & 0.090 & $6(13)$ & $14(9)$ & 0.429 \\
\hline 3 & $174(89)$ & $88(94)$ & $86(84)$ & $0.039^{*}$ & $38(84)$ & $136(90)$ & 0.294 \\
\hline \multicolumn{8}{|l|}{ Lesion location (n (\%)) } \\
\hline Left main stenosis & $78(40)$ & $36(38)$ & $42(41)$ & 0.770 & $26(58)$ & $52(34)$ & $0.005^{*}$ \\
\hline
\end{tabular}




\section{Continued}

LAD
RCA
CX
Characteristics

XCL time (minutes) (median, IQR)

$\mathrm{CPB}$ time (minutes) (median, IQR)

Graft per case (No. (n (\%)))

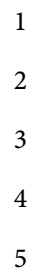

Graft usage (n (\%))

LIMA
RIMA
Vein graft
Radial artery

Reoperation (n (\%))

Postoperative renal dysfunction (n (\%))

Postoperative atrial fibrillation (n (\%))

Postoperative arrhythmia (n (\%))

Postoperative blockage (n (\%))

Length of stay in ICU (days) (median, IQR)

Length of stay in referring department (days) (median, IQR)

$\begin{array}{ll}192(98) & 93(99) \\ 183(96) & 91(97) \\ 188(96) & 91(97)\end{array}$

$99(97)$

$92(90)$

0.622

45 (100)

147 (97)

0.576

$92(90) \quad 0.063$

41 (91)

$142(94)$

0.500

$97(95)$

0.723

$43(96)$

$145(96)$

1.000

$\begin{array}{lllllll}46(35 ; 59) & 47(37 ; 59) & 46(32 ; 57) & 0.384 & 49(36 ; 62) & 45(35 ; 59) & 0.279 \\ 79(63 ; 99) & 80(66 ; 99) & 78(60 ; 99) & 0.611 & 92(65 ; 104) & 77(63 ; 95) & 0.061\end{array}$

$\begin{array}{ccccccc}* * & * * & * * & 1.000 & * * & * * & 0.230 \\ 48(25) & 16(17) & 32(31) & 0.020^{*} & 9(20) & 39(26) & 0.425 \\ 94(48) & 46(49) & 48(47) & 0.793 & 23(51) & 71(47) & 0.630 \\ 43(22) & 25(27) & 18(18) & 0.130 & 11(24) & 32(21) & 0.644 \\ 10(5) & 7(7) & * * & 0.200 & * * & 9(6) & 0.459\end{array}$

$\begin{array}{ccccccc}193(99) & 94(100) & 99(97) & 0.094 & 45(100) & 148(98) & 1.000 \\ 7(4) & 4(4) & * * & 0.712 & * * & 5(3) & 1.661 \\ 184(94) & 89(95) & 95(93) & 0.652 & 41(91) & 143(95) & 0.476 \\ 9(5) & 4(4) & 5(5) & 1.000 & * * & 7(5) & 1.000 \\ * * & * * & * * & 0.229 & * * & * * & 1.000 \\ 5(3) & * * & * * & 1.000 & * * & * * & 0.081 \\ 45(23) & 13(14) & 32(31) & 0.004^{*} & 45(23) & \text { N/A } & \text { N/A } \\ 4(2) & * * & * * & 0.619 & * * & * * & 0.552 \\ * * & * * & * * & 1.000 & * * & * * & 1.000 \\ 1(1 ; 1) & 1(1 ; 1) & 1(1 ; 1) & 0.663 & 1(1 ; 1) & 1(1 ; 1) & 0.348 \\ 6(5 ; 8) & 6(5 ; 8) & 6(5 ; 7) & 0.809 & 7(5 ; 8) & 6(5 ; 7) & 0.004^{*}\end{array}$

POAF = Postoperative atrial fibrillation. IQR = Interquartile range. $\mathrm{IHD}=$ Ischemic heart disease. $\mathrm{CNS}=$ Central nerve system. COPD $=$ Chronic obstructive pulmonary disease. NYHA $=$ New York Heart Association. $\mathrm{CCS}=$ Canadian Cardiovascular Society grading of angina pectoris. MI $=$ Myocardial infarction. $\mathrm{PCI}=$ Percutaneous coronary intervention. $\mathrm{EF}=$ Ejection fraction (\%). BMI = Body Mass Index. $\mathrm{LAD}=$ Left anterior descending. $\mathrm{CX}=\mathrm{Circumflex}$ artery. $\mathrm{RCA}=$ Right coronary artery. $\mathrm{XCL}$ time $=$ Aortic Cross-clamp time. $\mathrm{CPB}$ time $=$ Cardiopulmonary bypass time. LIMA $=$ Left internal mammary artery. RIMA $=$ Right internal mammary artery. ICU $=$ Intensive Care Unit. ${ }^{*}$ Three or fewer cases. ${ }^{*}$ Significant $p$-value $<0.05$. N/A $=$ Not applicable.

POAF were older, received placebo, more likely to have higher EuroSCORE, and left main stenosis compared to patients who stayed in sinus rhythm. Further, patients with POAF had a longer stay in referring department.

\subsection{Long-Term Side Effects}

Potential long-term side effects of amiodarone were evaluated based on registered diagnostic codes as described in the method section. When comparing the subcategories, we found no statistically significant difference between prophylactic groups. Furthermore, no statistically significant difference between prophylactic groups was found, when comparing the uncategorized diagnostic codes. 


\subsection{HARS and Long-Term Mortality}

There were no statistically significant differences between prophylactic groups regarding HARS in Table 2. When comparing subgroups, though, patients who developed POAF, were more likely to develop late AF. None of the other variables of HARS were statistically significant different between subgroups. Among the 195 patients included in the follow-up analysis, 36\% died during the 12-year follow-up. Of those, $39 \%$ died of cardiovascular diseases and $58 \%$ of others causes with no significant differences between the prophylactic groups. Cause of death was not registered in two patients (3\%). Patients who developed POAF, had a higher long-term all-cause mortality compared with those who stayed in sinus rhythm in Table 2.

\subsection{Factors Associated with Late AF, Readmission and Long-Term Mortality}

In both the uni- and multivariable Cox regression analysis, amiodarone was not statistically significant associated with an increased risk of readmission, late AF, long-term all-cause mortality or cardiovascular mortality in Table 3. In the univariable Cox regression analysis POAF was associated with a higher risk of late AF [95\% CI 2.28 1.15; 4.56], long-term cardiovascular mortality HR 2.34 [95\% CI 1.10; 5.00] and all-cause mortality HR 1.74 [95\% CI 1.05; 2.88], although not significant in the adjusted analysis in Table 4. POAF was not associated with a higher risk of readmission in Table 4.

Table 2. Follow-up data on heart failure, late AF, readmission, stroke and long-term all-cause mortality.

\begin{tabular}{|c|c|c|c|c|c|c|c|}
\hline & \multirow{2}{*}{ All } & \multicolumn{2}{|c|}{ Prophylactic Groups } & \multirow{2}{*}{$p$-Value ${ }^{*}$} & \multicolumn{2}{|c|}{ Subgroups } & \multirow{2}{*}{$p$-Value ${ }^{*}$} \\
\hline & & Amiodarone & Placebo & & POAF & NO POAF & \\
\hline \multicolumn{8}{|l|}{ Variables } \\
\hline Long term all-cause mortality (n (\%)) & $71(36)$ & $35(37)$ & $36(36)$ & 0.818 & $22(49)$ & $49(33)$ & $0.047^{*}$ \\
\hline Readmission (n (\%)) & $190(97)$ & $91(97)$ & $99(98)$ & 0.674 & $44(98)$ & $146(97)$ & 1.000 \\
\hline Late AF (n (\%)) & $34(17)$ & $21(22)$ & $13(13)$ & 0.082 & $13(29)$ & $21(14)$ & $0.020^{*}$ \\
\hline Stroke (n (\%)) & $25(13)$ & $12(12)$ & $13(14)$ & 0.684 & $9(20)$ & $16(11)$ & 0.100 \\
\hline Heart failure (n (\%)) & $22(11)$ & $12(13)$ & $10(10)$ & 0.527 & $5(11)$ & $17(11)$ & 0.967 \\
\hline
\end{tabular}

POAF $=$ Postoperative atrial fibrillation. $\mathrm{AF}=$ Atrial fibrillation. ${ }^{\star}$ Significant $p$-value $<0.05$.

Table 3. Unadjusted and adjusted Cox Proportional Hazard Regression Models Association between amiodarone and readmission, late AF, long-term cardiovascular mortality and all-cause mortality.

\begin{tabular}{|c|c|c|c|c|c|c|c|c|}
\hline & \multicolumn{2}{|c|}{ Readmission } & \multicolumn{2}{|c|}{ Late AF } & \multicolumn{4}{|c|}{ Long-Term Cardiovascular Mortality Long-Term All-Cause Mortality } \\
\hline & $\begin{array}{c}\text { Univariable } \\
\text { HR (95\% CI) }\end{array}$ & $\begin{array}{l}\text { Multivariable } \\
\text { HR (95\% CI) }\end{array}$ & $\begin{array}{c}\text { Univariable } \\
\text { HR }(95 \% \text { CI })\end{array}$ & $\begin{array}{l}\text { Multivariable }^{\mathrm{b}} \\
\text { HR }(95 \% \mathrm{CI})\end{array}$ & $\begin{array}{c}\text { Univariable } \\
\text { HR }(95 \% \mathrm{CI})\end{array}$ & $\begin{array}{l}\text { Multivariable }^{c} \\
\text { HR }(95 \% \text { CI })\end{array}$ & $\begin{array}{c}\text { Univariable } \\
\text { HR (95\% CI) }\end{array}$ & $\begin{array}{l}\text { Multivariable }^{a} \\
\text { HR }(95 \% \text { CI })\end{array}$ \\
\hline Amiodarone & $0.97(0.73 ; 1.29)$ & $0.94(0.69 ; 1.26)$ & $1.80(0.90 ; 3.59)$ & $2.01(0.99 ; 4.11)$ & $1.07(0.51 ; 2.43)$ & $1.30(0.61 ; 2.78)$ & $1.06(0.67 ; 1.69)$ & $0.96(0.59 ; 1.55)$ \\
\hline
\end{tabular}

$\mathrm{HR}=$ Hazard ratio. $\mathrm{CI}=$ Confidence interval. $\mathrm{POAF}=$ Postoperative atrial fibrillation. $\mathrm{AF}=$ Atrial fibrillation. ${ }^{\mathrm{a}} \mathrm{Adjusted}$ for age, $\mathrm{POAF}$ and $\mathrm{Vessels}$ affected 3 ; ${ }^{\mathrm{b}}$ Adjusted for age and POAF; ${ }^{\mathrm{c}}$ Adjusted for POAF. 


\subsection{Long-Term Survival}

Despite non-violation of proportional hazard assumption, the survival curves for the prophylactic groups crossed more than once in Figure 2(a).

Table 4. Unadjusted and Adjusted Cox Proportional Hazard Regression Models Association between POAF and readmission, late AF, long-term cardiovascular mortality and all-cause mortality

\begin{tabular}{|c|c|c|c|c|c|c|c|c|}
\hline & \multicolumn{2}{|c|}{ Readmission } & \multicolumn{2}{|c|}{ Late AF } & \multicolumn{2}{|c|}{ Long-Term Cardiovascular Mortality } & \multicolumn{2}{|c|}{ Long Term All-Cause Mortality } \\
\hline & $\begin{array}{c}\text { Univariable } \\
\text { HR }(95 \% \mathrm{CI})\end{array}$ & $\begin{array}{c}\text { Multivariable } \mathrm{e}^{\mathrm{a}} \\
\text { HR }(95 \% \mathrm{CI})\end{array}$ & $\begin{array}{c}\text { Univariable } \\
\text { HR }(95 \% \mathrm{CI})\end{array}$ & $\begin{array}{c}\text { Multivariable }{ }^{\mathrm{b}} \\
\text { HR }(95 \% \text { CI) }\end{array}$ & $\begin{array}{c}\text { Univariable } \\
\text { HR }(95 \% \mathrm{CI})\end{array}$ & $\begin{array}{c}\text { Multivariable }^{c} \\
\text { HR }(95 \% \text { CI) }\end{array}$ & $\begin{array}{c}\text { Univariable } \\
\text { HR (95\% CI) }\end{array}$ & $\begin{array}{c}\text { Multivariable }{ }^{\mathrm{a}} \\
\text { HR }(95 \% \mathrm{CI})\end{array}$ \\
\hline POAF & $\begin{array}{c}0.81(0.58 \\
1.14)\end{array}$ & $0.78(0.54 ; 1.12)$ & $\begin{array}{c}2.28(1.15 ; \\
4.56)\end{array}$ & $1.60(0.79 ; 3.25)$ & $2.34(1.10 ; 5.00)$ & $1.62(0.75 ; 3.51)$ & $1.74(1.05 ; 2.88)$ & $1.17(0.67 ; 2.04)$ \\
\hline
\end{tabular}

$\mathrm{HR}=$ Hazard ratio. $\mathrm{CI}=$ Confidence interval. POAF $=$ Postoperative atrial fibrillation. AF $=$ Atrial fibrillation. ${ }^{2}$ Adjusted for age, EuroSCORE, Left main stenosis, and Length of stay in referring department; ${ }^{\mathrm{b}}$ Adjusted for age, EuroSCORE; ${ }^{\mathrm{c} A d j u s t e d ~ f o r ~ a g e . ~}$

(a)

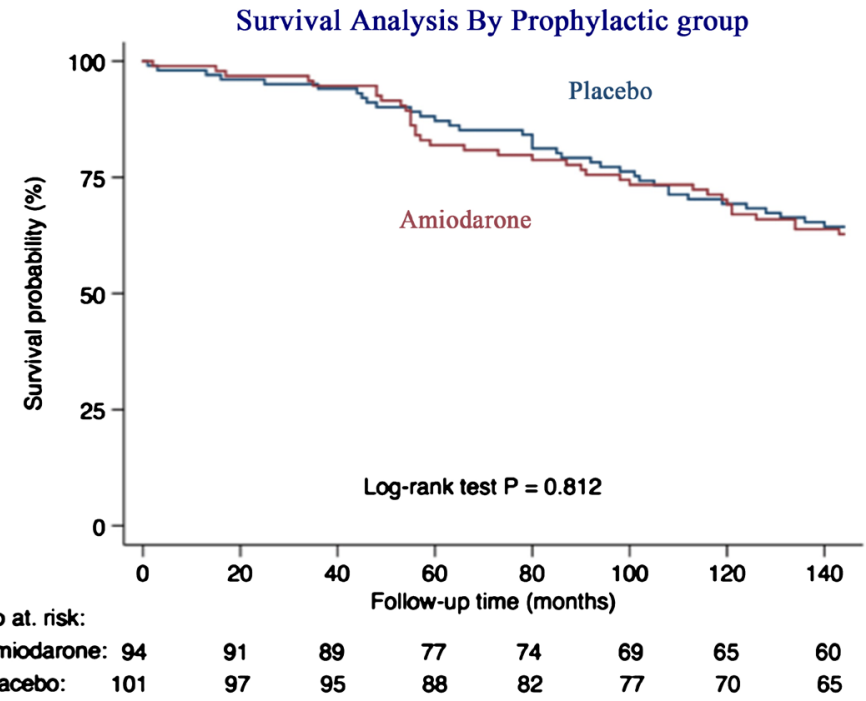

(b)

Survival Analysis By Subgroup

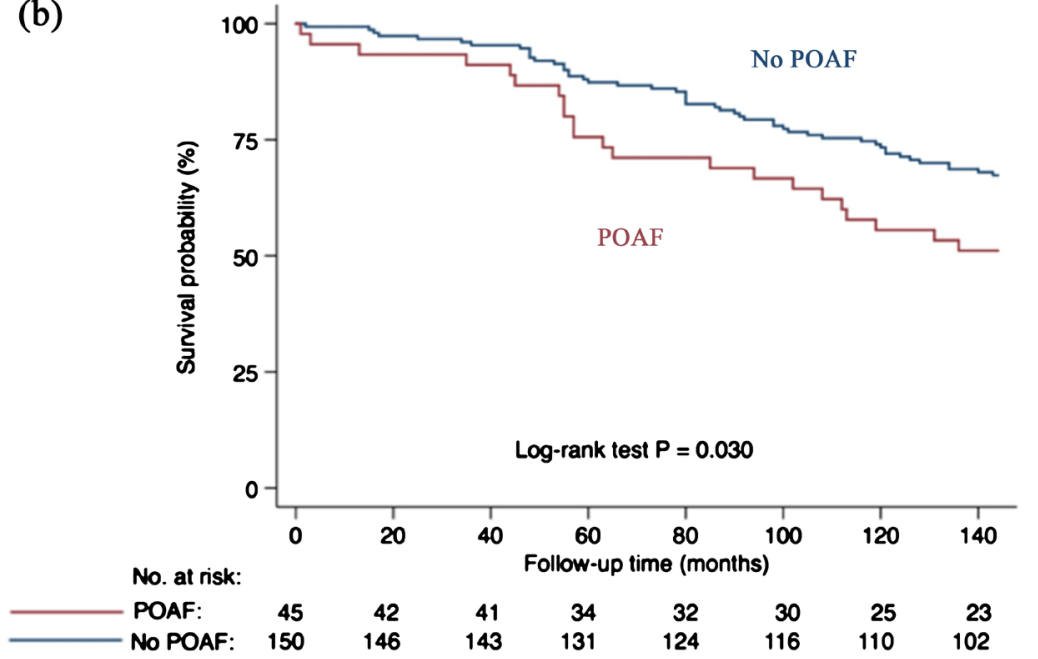

Figure 2. Kaplan Meier survival curves displaying long-term survival of patients undergoing coronary artery bypass grafting, stratified by prophylactic groups (a), and subgroups (b). Risk tables are displayed under the curves. POAF: postoperative atrial fibrillation. 
Since the overall survival of our cohort was greater than $50 \%$ at the last time point, we were not able to calculate the median survival time. When comparing the survival curves of the prophylactic groups, we found no statistically significant difference in the overall long-term survival in Figure 2(a). However, we did find a decreased overall long-term survival, in patients who developed POAF in Figure 2(b).

\subsection{Competing Risk Analysis}

We investigated the cumulative incidence function (CIF) of readmission and late $\mathrm{AF}$ with death as a possible competing risk within prophylactic groups (a) and subgroups (b), respectively in Figure 3 and Figure 4. No statistically significant differences in the risk of readmission were found. POAF was associated with an increased risk of late AF HR 2.28 [95\% CI 1.15; 4.55], when accounting for death as a competing risk. Amiodarone was not associated with late AF.
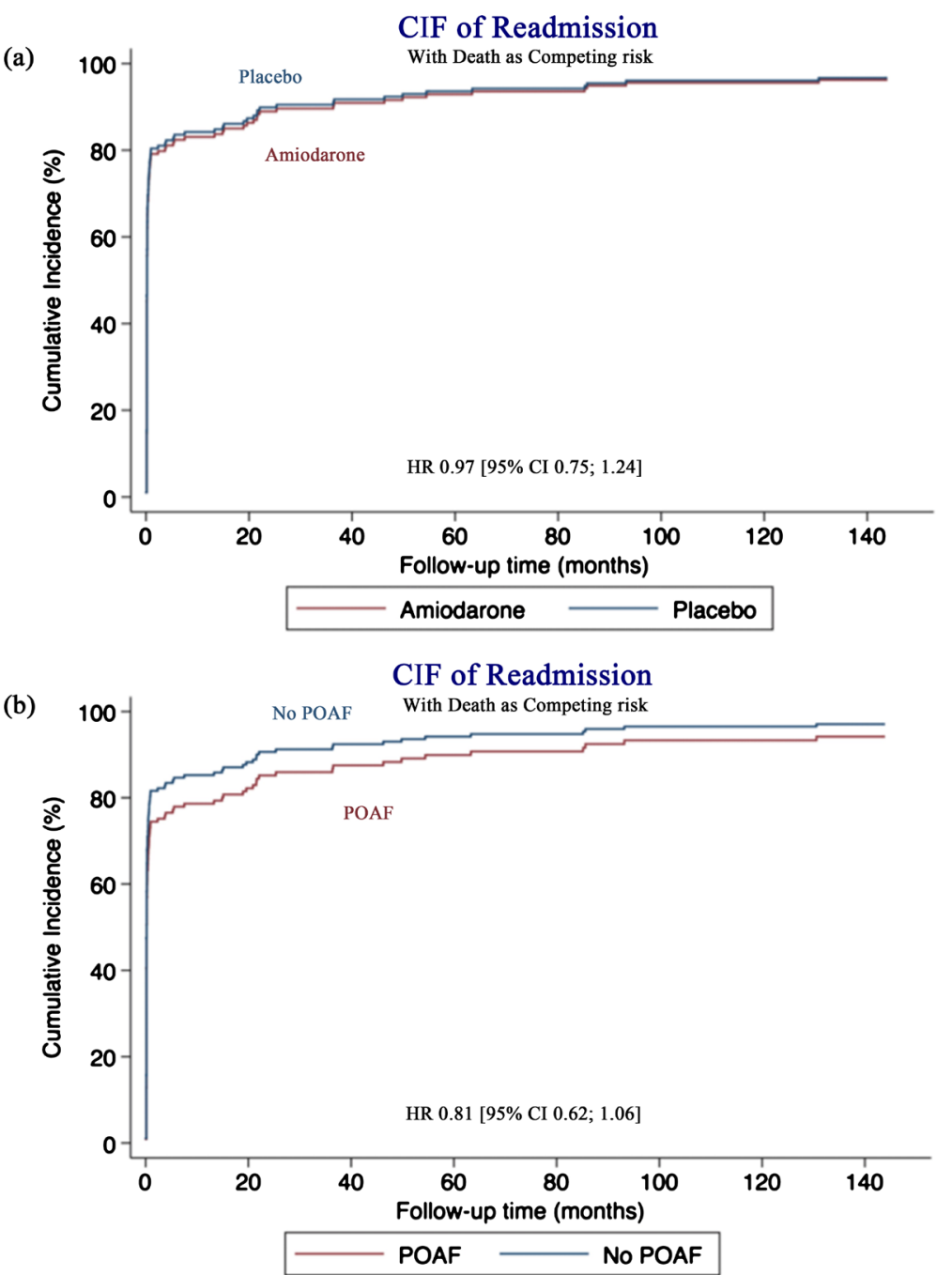

Figure 3. Cumulative incidence function (CIF) of readmission, with death as a competing risk, stratified by prophylactic groups (a), and subgroups (b). Competing risk regression was used to estimate subdistribution HR (SHR). POAF: postoperative atrial fibrillation. 


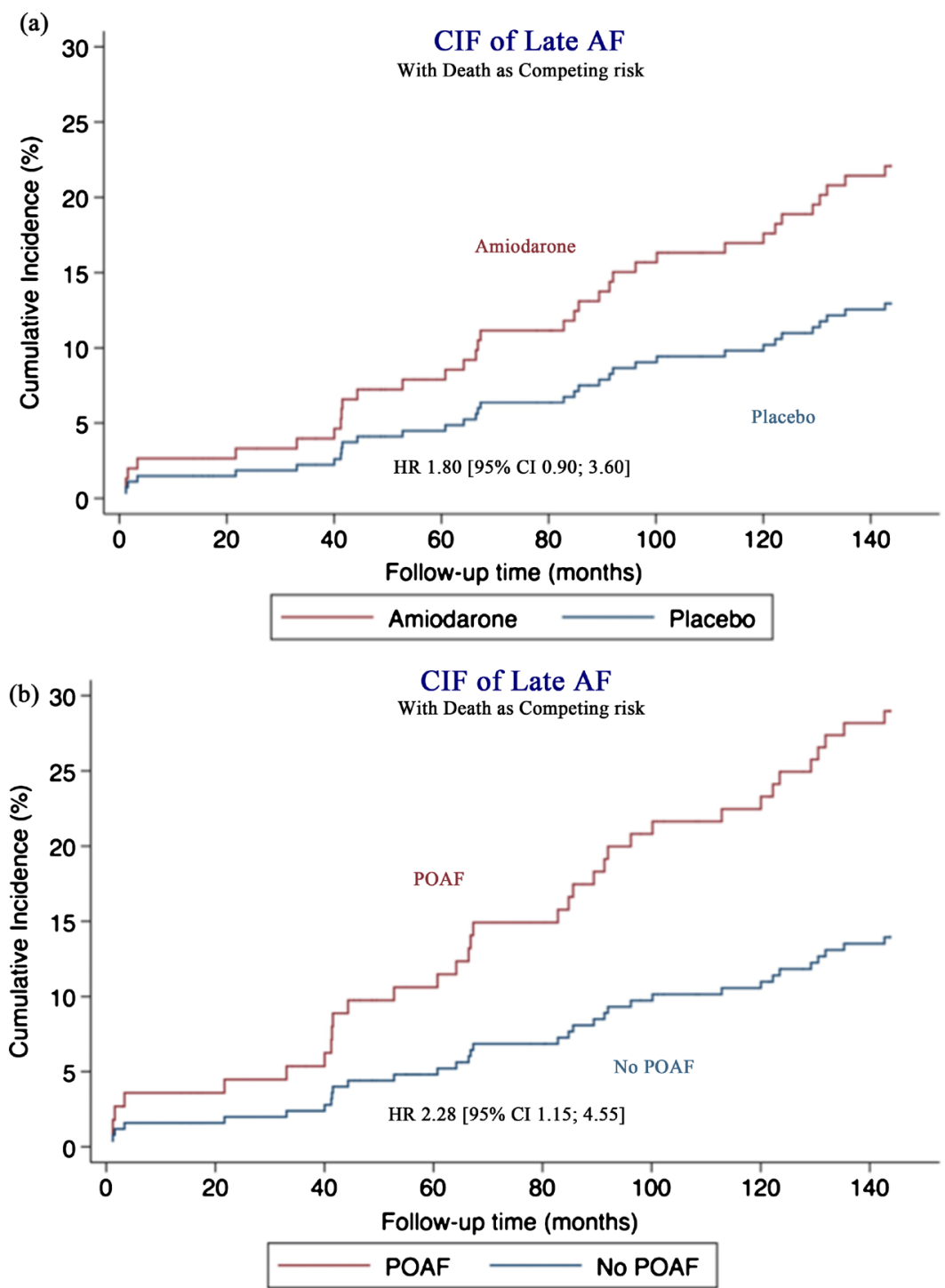

Figure 4. Cumulative incidence function (CIF) of late AF, with death as a competing risk, stratified by prophylactic groups (a), and subgroups (b). Competing risk regression was used to estimate subdistribution HR (SHR). POAF: postoperative atrial fibrillation.

\section{Discussion}

In this 12-year follow-up study, we found no differences in long-term side effects within the prophylactic groups. Furthermore, the long-term overall survival was not statistically significant different within prophylactic groups, and there was no association found between amiodarone and the risk of late $\mathrm{AF}$, readmission or long-term mortality, in the competing risk analysis or multivariable analysis. While no difference was found in the long-term overall survival between prophylactic groups, patients who developed POAF had a worse long-term overall survival, and were more likely to develop late AF, compared to patients who stayed in sinus rhythm. In the competing risk analysis, POAF was not associated with an increased risk of readmission. However, POAF was associated with an increased risk of late $\mathrm{AF}$, when accounting for death as a competing risk. In the 
multivariable analysis, no associations were found within subgroups regarding late $\mathrm{AF}$, readmission or long-term mortality.

The use of amiodarone has previously been associated with a number of short-term side effects and more serious adverse effects including skin cancer and toxicity of lung, thyroid and liver [17] [18] [19] [20] [21]. In this current study, we found no statistically significant increased risk for patients who had amiodarone prophylaxis in the RASCABG regime. Furthermore, we found no statistically significant difference in the risk of readmission, late AF or long-term mortality within prophylactic groups, indicating that amiodarone may be less hazardous than previous alleged [17] [18] [19] [20] [21].

When comparing subgroups, patients who developed POAF were more likely to develop late $\mathrm{AF}$ and die compared to patients who remained in sinus rhythm. This finding is consistent with studies investigating the association between POAF, late AF and long-term mortality [5] [22] [23] [24].

In current studies, POAF has been associated with an increased risk of late AF, and long-term mortality [5] [22] [23] [24]. Despite corresponding findings of the competing risk regression and survival analysis, POAF was not associated with an increased risk of late AF, and long-term mortality in the adjusted Cox regression analysis of our study. This might be due to inadequate power when adjusting for possible confounders.

\section{Strength and Limitations}

The main strengths of our study are the population, which were included from a double-blinded, randomized control trial, and the data source, as follow-up data were obtained from validated national databases. Additionally, the original RASCABG study was powered by the number of patients included, reflecting a verified sample size calculation, minimizing type II error. Naturally, some limitations should be noted. First, the study was based on data from single center study, which may limit the generalizability of the results, though, the surgical procedures were performed by 10 different cardiac surgeons. Second, some of the follow-up data were based on diagnostic codes and the validity of the results, therefore it depends on similar reporting. Third, as many other retrospective studies, our study contained missing data. In total, missing data were present in 20 baseline variables, with missing values ranging from $0.4 \%$ to $15.7 \%$.

\section{Conclusion}

Amiodarone was found to be a safe option in the prophylactic setting of RASCABG within a 12-year follow-up period and should be considered in patients undergoing isolated, elective CABG. POAF was found to be related with long-term worse prognosis regarding late $\mathrm{AF}$ and mortality.

\section{Funding Statement}

Odense University Hospital Pregraduate Foundation. 


\section{Conflicts of Interest}

The authors declare no conflicts of interest regarding the publication of this paper.

\section{References}

[1] Raiten, J.M., Ghadimi, K., Augoustides, J.G., Ramakrishna, H., Patel, P.A., Weiss, S.J., et al. (2015) Atrial Fibrillation after Cardiac Surgery: Clinical Update on Mechanisms and Prophylactic Strategies. Journal of Cardiothoracic and Vascular Anesthesia, 29, 806-816. https://doi.org/10.1053/j.jvca.2015.01.001

[2] Bessissow, A., Khan, J., Devereaux, P.J., Alvarez-Garcia, J. and Alonso-Coello, P. (2015) Postoperative Atrial Fibrillation in Non-Cardiac and Cardiac Surgery: An Overview. Journal of Thrombosis and Haemostasis, 13, 304-312. https://doi.org/10.1111/jth.12974

[3] Greenberg, J.W., Lancaster, T.S., Schuessler, R.B. and Melby, S.J. (2017) Postoperative Atrial Fibrillation Following Cardiac Surgery: A Persistent Complication. European Journal of Cardio- Thoracic Surgery, 52, 665-672. https://doi.org/10.1093/ejcts/ezx039

[4] Yadava, M., Hughey, A.B. and Crawford, T.C. (2016) Postoperative Atrial Fibrillation: Incidence, Mechanisms, and Clinical Correlates. Heart Failure Clinics, 12, 299-308. https://doi.org/10.1016/j.hfc.2015.08.023

[5] Phan, K., Ha, H.S., Phan, S., Medi, C., Thomas, S.P. and Yan, T.D. (2015) New-Onset Atrial Fibrillation Following Coronary Bypass Surgery Predicts Long-Term Mortality: A Systematic Review and Meta-Analysis. European Journal of Cardio-Thoracic Surgery, 48, 817-824. https://doi.org/10.1093/ejcts/ezu551

[6] Megens, M.R., Churilov, L. and Thijs, V. (2017) New-Onset Atrial Fibrillation after Coronary Artery Bypass Graft and Long-Term Risk of Stroke: A Meta-Analysis. Journal of the American Heart Association, 6, e007558. https://doi.org/10.1161/JAHA.117.007558

[7] Tulla, H., Hippelainen, M., Turpeinen, A., Pitkanen, O. and Hartikainen, J. (2015) New-Onset Atrial Fibrillation at Discharge in Patients after Coronary Artery Bypass Surgery: Short- and Long-Term Morbidity and Mortality. European Journal of Cardio-Thoracic Surgery, 48, 747-752. https://doi.org/10.1093/ejcts/ezu526

[8] Alawami, M., Chatfield, A., Ghashi, R. and Walker, L. (2018) Atrial Fibrillation after Cardiac Surgery: Prevention and Management: The Australasian Experience. Journal of the Saudi Heart Association, 30, 40-46.

https://doi.org/10.1016/j.jsha.2017.03.008

[9] Thein, P.M., White, K., Banker, K., Lunny, C., Mirzaee, S. and Nasis, A. (2018) Preoperative Use of Oral Beta-Adrenergic Blocking Agents and the Incidence of New-Onset Atrial Fibrillation after Cardiac Surgery. A Systematic Review and Meta-Analysis. Heart, Lung and Circulation, 27, 310-321. https://doi.org/10.1016/j.hlc.2017.08.026

[10] Lomivorotov, V.V., Efremov, S.M., Pokushalov, E.A. and Karaskov, A.M. (2016) New-Onset Atrial Fibrillation after Cardiac Surgery: Pathophysiology, Prophylaxis, and Treatment. Journal of Cardiothoracic and Vascular Anesthesia, 30, 200-216. https://doi.org/10.1053/j.jvca.2015.08.003

[11] Kirchhof, P., Benussi, S., Kotecha, D., Ahlsson, A., Atar, D., Casadei, B., et al. (2016) ESC Guidelines for the Management of Atrial Fibrillation Developed in Collaboration with EACTS. European Journal of Cardio- Thoracic Surgery, 50, 2893-2962. 
https://doi.org/10.1093/ejcts/ezw313

[12] Zebis, L.R., Christensen, T.D., Thomsen, H.F., Mikkelsen, M.M., Folkersen, L., Sorensen, H.T., et al. (2007) Practical Regimen for Amiodarone Use in Preventing Postoperative Atrial Fibrillation. The Annals of Thoracic Surgery, 83, 1326-1331. https://doi.org/10.1016/j.athoracsur.2006.09.096

[13] Schmidt, M., Maeng, M., Jakobsen, C.J., Madsen, M., Thuesen, L., Nielsen, P.H., et al. (2010) Existing Data Sources for Clinical Epidemiology: The Western Denmark Heart Registry. Clinical Epidemiology, 2, 137-144. https://doi.org/10.2147/CLEP.S10190

[14] Lynge, E., Sandegaard, J.L. and Rebolj, M. (2011) The Danish National Patient Register. Scandinavian Journal of Public Health, 39, 30-33. https://doi.org/10.1177/1403494811401482

[15] Helweg-Larsen, K. (2011) The Danish Register of Causes of Death. Scandinavian Journal of Public Health, 39, 26-29. https://doi.org/10.1177/1403494811399958

[16] Fine, J. and Gray, R. (1999) A Proportional Hazards Model for the Subdistribution of a Competing Risk. Journal of the American Statistical Association, 94, 496-509. https://doi.org/10.1080/01621459.1999.10474144

[17] Teerakanok, J., Tantrachoti, P., Chariyawong, P. and Nugent, K. (2016) Acute Amiodarone Pulmonary Toxicity after Surgical Procedures. The American Journal of the Medical Sciences, 352, 646-651. https://doi.org/10.1016/j.amjms.2016.08.013

[18] Jackevicius, C.A., Tom, A., Essebag, V., Eisenberg, M.J., Rahme, E., Tu, J.V., et al. (2011) Population-Level Incidence and Risk Factors for Pulmonary Toxicity Associated with Amiodarone. American Journal of Cardiology, 108, 705-710. https://doi.org/10.1016/j.amjcard.2011.04.024

[19] Trohman, R.G., Sharma, P.S., McAninch, E.A. and Bianco, A.C. (2018) Amiodarone and the Thyroid Physiology, Pathophysiology, Diagnosis and Management. Trends in Cardiovascular Medicine, 29, 285-295. https://doi.org/10.1016/j.tcm.2018.09.005

[20] Hashmi, A., Keswani, N.R., Kim, S. and Graham, D.Y. (2016) Hepatic Dysfunction in Patients Receiving Intravenous Amiodarone. The Southern Medical Journal, 109, 83-86. https://doi.org/10.14423/SMJ.0000000000000413

[21] Maoz, K.B., Dvash, S., Brenner, S. and Brenner, S. (2009) Amiodarone-Induced Skin Pigmentation and Multiple Basal-Cell Carcinomas. International Journal of Dermatology, 48, 1398-1400. https://doi.org/10.1111/j.1365-4632.2008.03819.x

[22] Lee, S.H., Kang, D.R., Uhm, J.S., Shim, J., Sung, J.H., Kim, J.Y., et al. (2014) New-Onset Atrial Fibrillation Predicts Long-Term Newly Developed Atrial Fibrillation after Coronary Artery Bypass Graft. American Heart Journal, 167, 593-600. https://doi.org/10.1016/j.ahj.2013.12.010

[23] Lowres, N., Mulcahy, G., Jin, K., Gallagher, R., Neubeck, L. and Freedman, B. (2018) Incidence of Postoperative Atrial Fibrillation Recurrence in Patients Discharged in Sinus Rhythm after Cardiac Surgery: A Systematic Review and Meta-Analysis. Interactive Cardio Vascular and Thoracic Surgery, 26, 504-511. https://doi.org/10.1093/icvts/ivx348

[24] Pillarisetti, J., Patel, A., Bommana, S., Guda, R., Falbe, J., Zorn, G.T., et al. (2014) Atrial Fibrillation Following Open Heart Surgery: Long-Term Incidence and Prognosis. Journal of Interventional Cardiac Electrophysiology, 39, 69-75. https://doi.org/10.1007/s10840-013-9830-6 